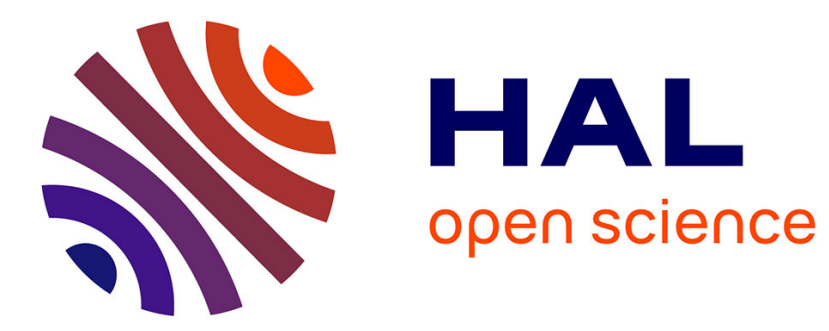

\title{
Dissecting the role of the sergeants in supramolecular helical catalysts: from chain capping to intercalation
}

Mayte A. Martínez-Aguirre, Yan Li, Nicolas Vanthuyne, Laurent Bouteiller, Matthieu Raynal

\section{- To cite this version:}

Mayte A. Martínez-Aguirre, Yan Li, Nicolas Vanthuyne, Laurent Bouteiller, Matthieu Raynal. Dissecting the role of the sergeants in supramolecular helical catalysts: from chain capping to intercalation. Angewandte Chemie International Edition, 2021, 60, pp.4183-4191. 10.1002/anie.202012457 . hal-03009425

\section{HAL Id: hal-03009425 \\ https://hal.sorbonne-universite.fr/hal-03009425}

Submitted on 17 Nov 2020

HAL is a multi-disciplinary open access archive for the deposit and dissemination of scientific research documents, whether they are published or not. The documents may come from teaching and research institutions in France or abroad, or from public or private research centers.
L'archive ouverte pluridisciplinaire HAL, est destinée au dépôt et à la diffusion de documents scientifiques de niveau recherche, publiés ou non, émanant des établissements d'enseignement et de recherche français ou étrangers, des laboratoires publics ou privés. 


\title{
Dissecting the role of the sergeants in supramolecular helical catalysts: from chain capping to intercalation
}

\author{
Mayte A. Martínez-Aguirre, ${ }^{\dagger[a]}$ Yan $L i,{ }^{\dagger[a]}$ Nicolas Vanthuyne,${ }^{[b]}$ Laurent Bouteiller, ${ }^{[a]}$ and Matthieu \\ Raynal ${ }^{*[a]}$ \\ [a] Mayte A. Martínez-Aguirre, Yan Li, Laurent Bouteiller, and Matthieu Raynal \\ Sorbonne Université, CNRS, Institut Parisien de Chimie Moléculaire \\ Equipe Chimie des Polymères \\ 4 Place Jussieu, 75005 Paris (France) \\ ${ }^{\dagger}$ These authors contributed equally to the work. \\ E-mail : matthieu.raynal@sorbonne-universite.fr. \\ [b] Aix Marseille Université, Centrale Marseille \\ CNRS, iSm2, UMR 7313 \\ 13397 Marseille Cedex 20, France \\ Supporting information for this article is given via a link at the end of the document
}

\begin{abstract}
Controlling the properties of supramolecular assemblies requires unveiling the specific interactions between their components. In the present work, the catalytic properties and structure of co-assemblies composed of a benzene-1,3,5tricarboxamide (BTA) ligand coordinated to copper (the soldier) and seven enantiopure BTAs (the sergeants) have been determined. Whatever the sergeant, the enantioselectivity of the reaction is directly proportional to the optical purity of the supramolecular helices. More strikingly, the role played by the sergeant in the coassembly process differs significantly: from almost pure intercalator (when it is incorporated in the stacks of the soldier and generates long homochiral helices) to pure chain capper (when it leads to the formation of partly helically biased and short assemblies). The former situation leads to optimal enantioselectivity for the catalytic system under study $(58 \%$ ee) while the latter situation leads to very low selectivity $(8 \% \mathrm{ee})$. The successful rationalization of this high and unexpected difference is crucial for the development of more efficient catalysts and more elaborate supramolecular systems.
\end{abstract}

\section{Introduction}

In 1999, Green and co-workers reported the ability of a little amount of chiral monomers to dictate their stereochemical preference to a large number of achiral monomers in poly(isocyanates), ${ }^{[1]}$ a class of polymers adopting a dynamic helical configuration in solution. ${ }^{[2]}$ Since then, the so-called sergeants-and-soldiers (S\&S) effect has been found to be operative in a wide range of helical polymers ${ }^{[3]}$ as well as in supramolecular assemblies. ${ }^{[4]}$ In the latter case, amplification of chirality through the $S \& S$ effect provides a unique avenue towards the formation of single handed supramolecular helices. Mixing achiral and enantiopure complementary monomers emerged as a highly suitable method to generate functional coassemblies serving as templates for achiral gold complexes ${ }^{[5]}$ or nanoparticles, ${ }^{[6]}$ as CPL-emitting devices ${ }^{[7]}$ and as asymmetric catalysts. ${ }^{[8]}$

Disk-like molecules decorated with hydrogen bonding moieties, such as benzene-1,3,5-tricarboxamides (BTAs) and their derivatives, ${ }^{[9]}$ are unique in regards to their ability to form remarkably stable supramolecular helices by a combination of hydrogen bonding and aromatic interactions. It is classically established in these helical systems that soldiers and sergeants co-assemble through a cooperative mechanism ${ }^{[10]}$ yielding very long one-dimensional helices which are one-handed at the condition that a few percent of sergeants are present in the coassemblies. ${ }^{[8,}$, 11] We previously investigated helical coassemblies embedding achiral BTA ligands (coordinated to $\mathrm{Rh}^{[8 \mathrm{a}]}$ or $\left.\mathrm{Cu}\right)^{[8 \mathrm{~b}]}$ and enantiopure BTAs as asymmetric catalysts. In these systems, the enantioselectivity displayed by the catalyst is directly due to a high helical purity and length of the coassemblies. ${ }^{[8 c]}$ However, no guiding rules exist for the selection of appropriate sergeants: the only strategy available up to now was to evaluate a set of arbitrarily chosen enantiopure BTAs in a catalytic reaction of reference and select the sergeants that provided the highest selectivity for further studies. $\left.{ }^{[8 a,} 8 b\right]$ Interestingly, recent studies on multicomponent assemblies have revealed that the role of the sergeant might not be restricted to its ability to favour a preferred helical configuration. ${ }^{[12]}$ Indeed, specific interactions between the components during the coassembly process influences not only the composition ${ }^{[13]}$ of the assemblies, but also the sequence ${ }^{[14]}$ of the monomers and the length of the polymer chains. ${ }^{[15]}$ Finely modulating the structure of the co-assemblies is a particularly intriguing topic ${ }^{[16]}$ that should have strong consequences on the catalytic function of supramolecular polymers. Herein, we show that the drastic effect exerted by the nature of the sergeant on the selectivity of the catalytic reaction is rationalized by accurately probing the structure of the corresponding co-assemblies. Seven S\&S-type co-assemblies embedding different types of sergeants were evaluated in catalysis and characterized by circular dichroism (CD), UV-Vis absorption, Fourier-Transform Infrared (FT-IR) and Small-Angle Neutron Scattering (SANS) analyses. The efficiency of the sergeant can be delineated through its ability to act as a chain capper, to intercalate in the co-assemblies, or to play both roles.

\section{Results and Discussion}

Selection of the different sergeants 


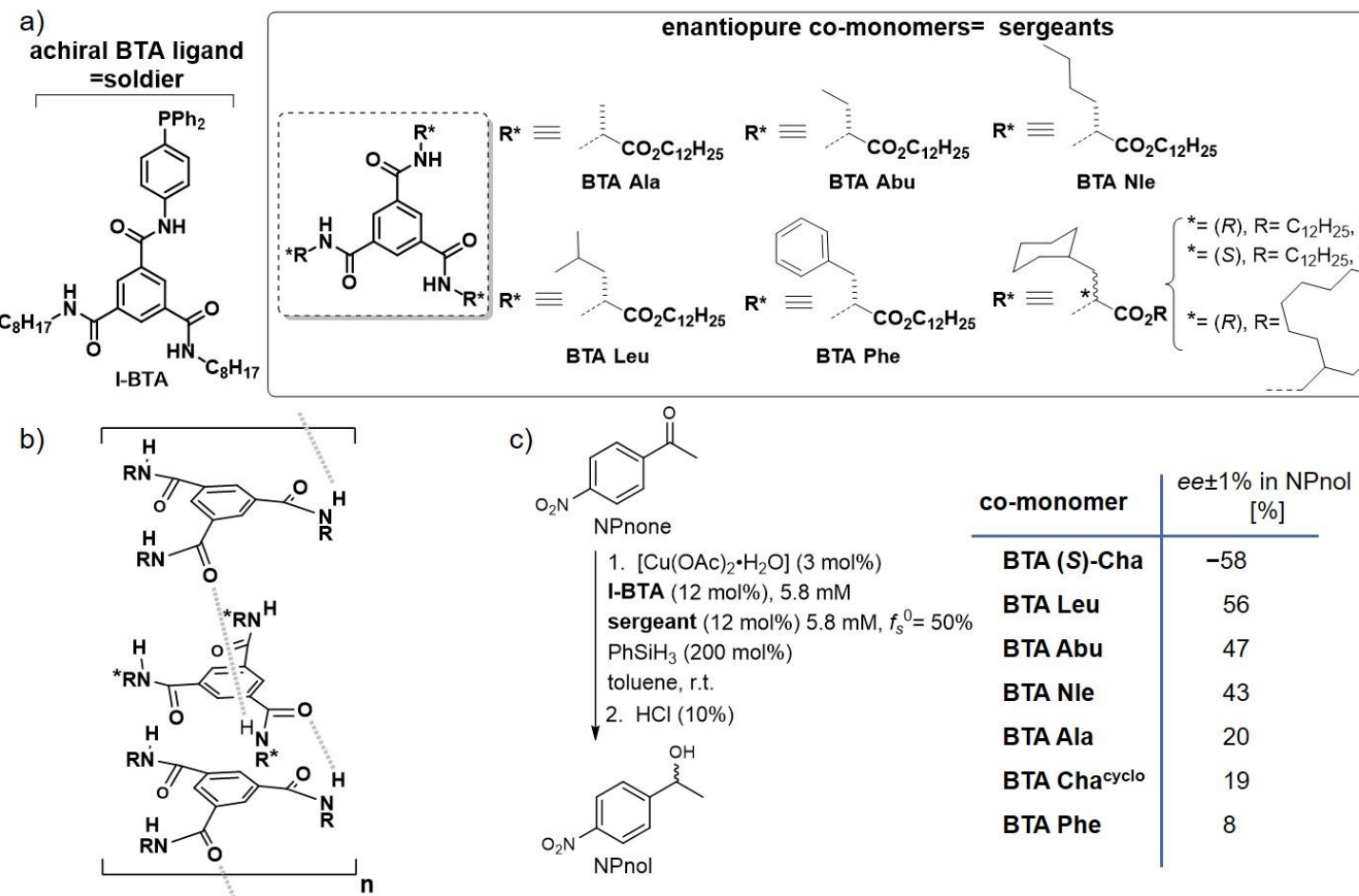

Scheme 1 a) Chemical structures of the BTA ligand and ester BTAs whose co-assembly properties have been studied in this manuscript. b) Schematic representation of the helical co-assemblies formed by the BTA ligand (the soldier) and the ester BTAs (the sergeant). Only one hydrogen bond, over the three established between consecutive monomers in the helical stacks, is shown for clarity. c) Screening of the sergeants in the copper-catalyzed hydrosilylation of NPnone into NPnol (overall fraction of sergeant $\left.=\mathrm{f}_{\mathrm{s}}{ }^{0}=50 \%\right)$. Positive (negative) ee values correspond to $(S)$-NPnol $((R)$-NPnol, respectively) being the major enantiomer. For the complete screening results see Table S1.

BTAs derived from $\alpha$-amino esters (ester BTAs) are a particularly interesting class of enantiopure BTAs given the high modularity of their molecular structure ${ }^{[17]}$ We decided to test a range of ester BTAs as sergeant to probe the influence of two design elements: the amino acid and the alkyl substituent. Each of these enantiopure ester BTAs was mixed with an achiral ligand (I-BTA) and tested in the copper-catalysed hydrosilylation of NPnone (Table S1). Based on this screening, we have selected six of them based on their selectivity levels (Scheme 1C $e e=$ enantiomeric excess): low $(8 \%<e e<20 \%$, BTA Phe and BTA Ala), moderate $(43 \%<e e<47 \%$, BTA Nle and BTA Abu) and high $(56 \%<|e e|<58 \%$, BTA Leu and BTA (S)-Cha). These six ester BTAs differ only in the nature of the amino acid fragment. In order to evaluate the impact of the bulkiness of the ester chain on the selectivity of the catalytic reaction, an analogue of BTA Cha with a methyl cyclododecyl group connected to the ester side chain was synthesized according to well established protocols (Supporting Information). BTA Cha $^{\text {cyclo }}$ was isolated pure predominantly as a single enantiomer (ee> 99\%, 96\% de) according to chiral HPLC analyses. Its evaluation as a sergeant in our catalytic reaction reveals that BTA Cha ${ }^{\text {cyclo }}$ provides a level of selectivity $(19 \%$ ee, Scheme 1c) far lower than BTA (S)-Cha, its analogue with a linear dodecyl ester side chain

\section{Combined catalytic experiments and CD studies}

In order to apprehend the origin of the different enantioselectivities observed in the catalytic reaction (Scheme 1c), mixtures containing I-BTA (concentration set constant to
$5.80 \mathrm{mM})$, the copper source $\left(\left[\mathrm{Cu}(\mathrm{OAc})_{2} \cdot \mathrm{H}_{2} \mathrm{O}\right], 1.45 \mathrm{mM}\right)$, and a sergeant in various amounts $\left(2.5 \% \leq \mathrm{fs}^{0} \leq 65 \%\right)$ have been analyzed by $\mathrm{CD}$ and engaged in the copper-catalysed hydrosilylation of NPnone. ${ }^{[18]}$ All solutions prepared during this study were homogeneous and both catalytic reactions and CD analyses were conducted under the same conditions providing remarkably reproducible results in terms of selectivities $( \pm 1 \%$ ee) and CD intensities $\left( \pm 0.2 \times 10^{-4} \mathrm{~g}\right.$ values). For all mixtures, a CD signal centered at $\approx 295 \mathrm{~nm}$ is present with virtually identical shapes whatever the nature of the sergeant (Figures 1, S1 and S2). This signal is absent from solutions containing only the soldier (because it is achiral) or containing only the sergeant (because it has no absorption band in this region). Such an induced $C D$ signal (ICD) is indicative of the extent of chirality induction of the soldier by the sergeant. ${ }^{[8 \mathrm{e}]}$ The corresponding $\mathrm{CD}$ intensities, under the form of the Kuhn anisotropy factor $\left(\mathrm{g}^{295}\right.$, see the caption of Figure 1), offer the possibility to compare the degree of helicity of the different co-assemblies.

The CD and UV-Vis absorption analyses of the different mixtures are shown in Figures 1, S1 and S2. Those concerning BTA Leu, BTA Nle and BTA Phe as sergeants are examined into more details in the following as they are representative of the trend exhibited by the other sergeants (vide infra). For BTA Leu, the CD intensity increases gradually until the fraction of sergeant is $\approx 40 \%$ and then reaches a plateau corresponding to a $g$ value of $7.7 \times 10^{-4}$ (Figure $1 b$ ) close to the one recorded for the best $S \& S$-type mixtures $\left(g_{\max } \approx 7.0 \times 10^{-4}\right) \cdot{ }^{[8]}$ This value is considered as the maximal value that can be reached for this type of helical assemblies and will be referred to as the homochiral state in the following discussion. Not only the fact 
that the maximal $g$ value is reached, but also the sharpening of the CD band are
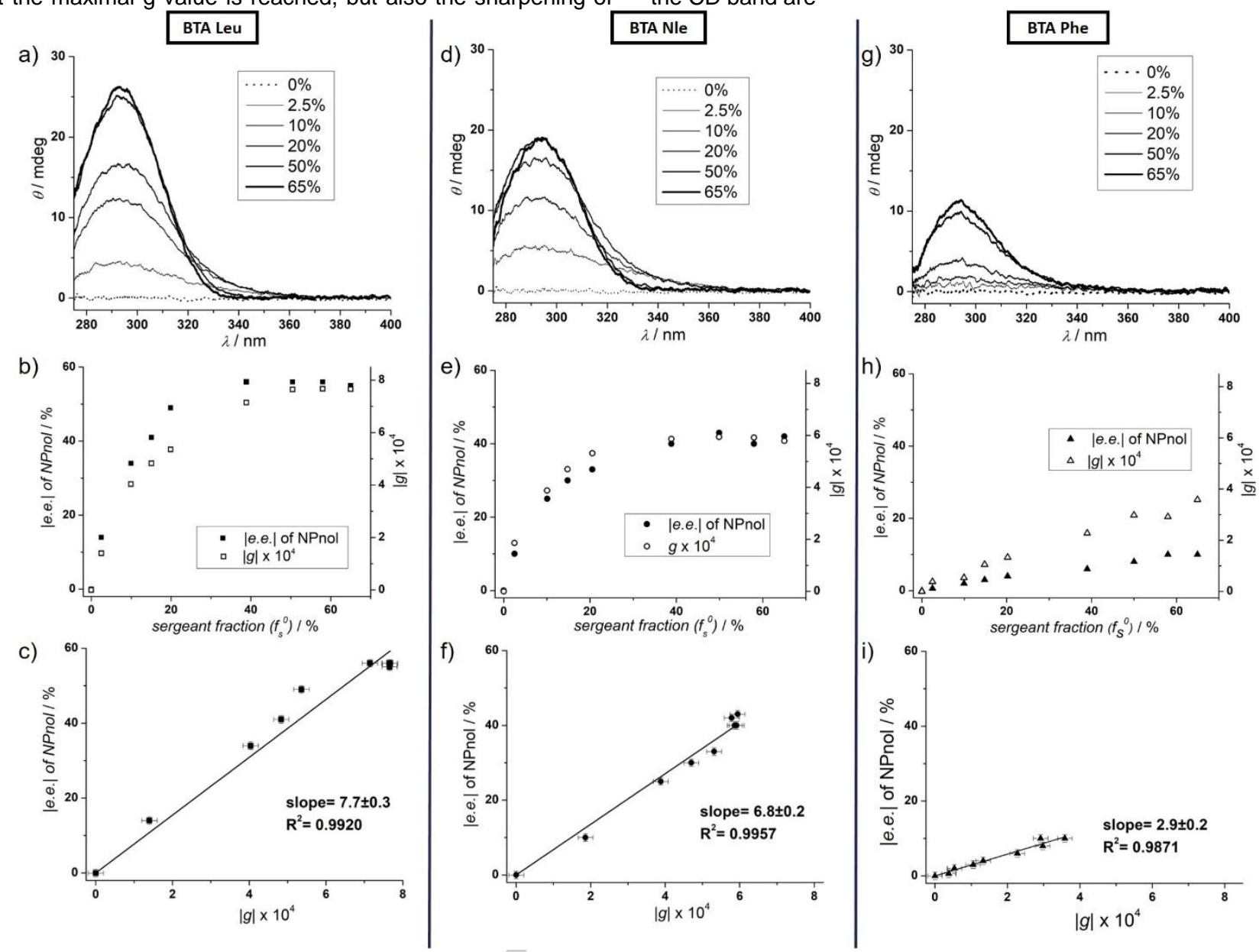

Figure 1 Correlation between helicity and enantioselectivity $\left(293 \mathrm{~K}\right.$, toluene, $\left.[\mathrm{Cu}]=\left[\mathrm{Cu}(\mathrm{OAC})_{2} \cdot \mathrm{H}_{2} \mathrm{O}\right]\right)$. (a, d, g) $\mathrm{CD}$ analyses of the sergeants-and-soldiers type mixtures between I-BTA, [Cu], and BTA Leu, BTA Nle or BTA Phe. Conditions: [I-BTA] $=5.80 \mathrm{mM}$, [I-BTA]/[Cu]= 4 and $0.15 \mathrm{mM}<[\mathrm{sergeant}]<10.8 \mathrm{mM}$ i.e. $2.5 \% \leq \mathrm{f}_{\mathrm{s}}{ }^{0} \leq 65 \%$. CD spectra for $\mathrm{f}_{\mathrm{s}}{ }^{0}=15 \%, \mathrm{f}_{\mathrm{s}}{ }^{0}=39 \%$ and $\mathrm{f}_{\mathrm{s}}{ }^{0}=58 \%$ are not shown for clarity. See Figures S1 and S2 for the UV-Vis absorption spectra. (b, e, $\mathbf{h}$ ) Plot of the enantioselectivity in NPnol versus the overall fraction of sergeant $\left(\mathrm{f}_{\mathrm{s}}{ }^{\circ}\right)$ in the mixtures. Conditions for the catalytic reactions: I-BTA (5.8 $\left.\mathrm{mM}, 12 \mathrm{~mol} \%\right)$, [Cu] $(1.45 \mathrm{mM}, 3 \mathrm{~mol} \%)$, sergeant $\left(2.5 \% \leq \mathrm{f}_{\mathrm{s}}{ }^{0} \leq 65 \%\right)$, NPnone $(48 \mathrm{mM}), \mathrm{PhSiH}_{3}(96 \mathrm{mM}), 293 \mathrm{~K}$. Conversion> $99 \%$ was obtained for all catalytic experiments, as determined by GC and ${ }^{1} \mathrm{H}$ NMR analyses. The optical purity was determined by GC analysis; all $(R)$-configured sergeants provide $(S)$-NPnol as the major enantiomer whilst BTA (S)-Cha yields $(R)$-NPnol. (c, $\mathbf{f}, \mathbf{i})$ Plot of the enantioselectivity in NPnol versus the Kuhn anisotropy factor. $\mathrm{g}^{295}=\theta^{295} /\left(32980 \times \mathrm{Abs}^{295}\right.$ ) where $\theta^{295}$ and $\mathrm{Abs}^{295}$ are the ellipticity and UV-Vis absorbance measured at $\lambda=295 \mathrm{~nm}$, respectively. Linear fitting is performed with a zero intercept fixed at origin.

indicative of the generation of homochiral helices upon increasing the fraction of sergeant in these mixtures. ${ }^{[8 \mathrm{e}]}$ Analyses of the catalytic data reveal that the selectivity is also maximal for $f_{s}{ }^{0} \geq 40 \%\left(e e_{\max } \approx 56 \%\right.$, Figure $\left.1 b\right)$. The similar trend exhibited by the selectivity and the degree of helicity as a function of $\mathrm{fs}_{\mathrm{s}}{ }^{0}$ is consistent with our precedent observations. ${ }^{[8 a, 8 e]}$ Plotting the selectivity in NPnol as a function of $g$ values actually reveals that the correlation between these two properties of the supramolecular helices is quantitative (Figure 1c). A similar trend is observed for S\&S-type mixtures containing BTA Nle as a sergeant with the notable exception that selectivity and $C D$ intensity level off at slightly lower values $\left(g_{\max } \approx 6.0 \times 10^{-4}, e e_{\max } \approx\right.$ $46 \%$, Figures $1 \mathrm{~d}-\mathrm{e})$. Selectivity of the catalytic reaction and optical purity of the helices are also linearly correlated, with a slope which is similar to that obtained for BTA Leu containing mixtures (Figure 1f). S\&S-type mixtures with BTA Phe exhibit more obvious differences because maximal $C D$ intensities and $g$ values are lower $\left(g_{\max } \approx 3.0 \times 10^{-4}\right.$, ee $\left.\max \approx 10 \%\right)$, and $g$ values increase more rapidly than ee values (Figures 1g-h). Even though a linear correlation is still observed between selectivity and optical purity (Figure 1i), the latter point translates into a more than two times lower slope comparatively to BTA Leu and BTA Nle S\&S-type mixtures.

The result of the combined spectroscopic/catalytic study for all the sergeants is shown in Figure $2 \mathrm{a}$ and $2 \mathrm{~b}$. First of all, it is important to note that the linear correlation between the selectivity in NPnol (from $0 \%$ ee to $e e_{\max }$ ) and the optical purity of the helices remains valid whatever the sergeant thus highlighting the intimate relationship between enantioselectivity and helicity in these systems. Three main classes of sergeants can be delineated: BTA Cha behaves as BTA Leu and allows the formation of homochiral helices $\left(g_{\max }\right)$ which in turn provides the maximal selectivity $\left(e e_{\max }\right)$ in the catalytic reaction of reference (class a), BTA Abu is similar to BTA Nle in its ability to promote almost (but not fully) optimal $g$ and ee values (class b), BTA Cha ${ }^{\text {cyclo }}$ and BTA Ala, similarly to BTA Phe, fail to produce efficient helical catalysts (class $c$ ). The distinction between efficient sergeants (classes $a$ and $b$ ) and poor ones 
(class $c$ ) is already observed for mixtures with a low amount of sergeant indicating that this sorting is valid whatever the feeding

ratio (Figure S3).
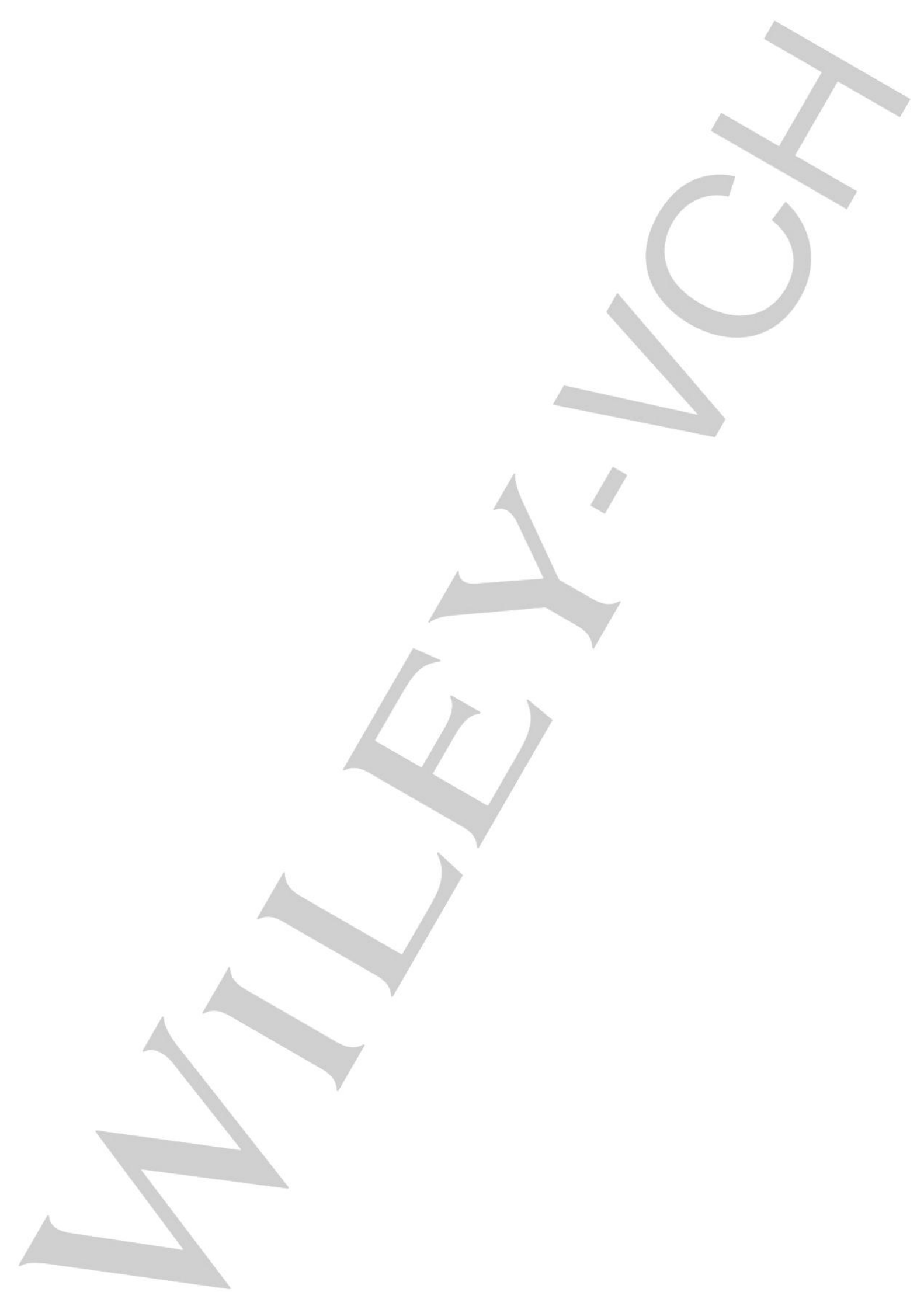
a)

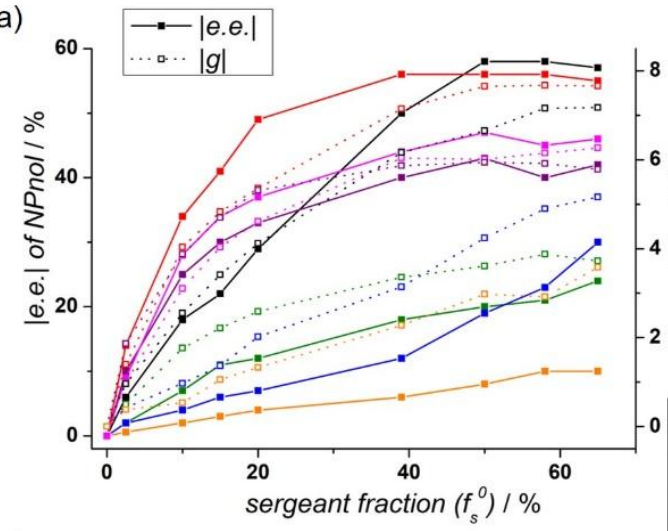

c)

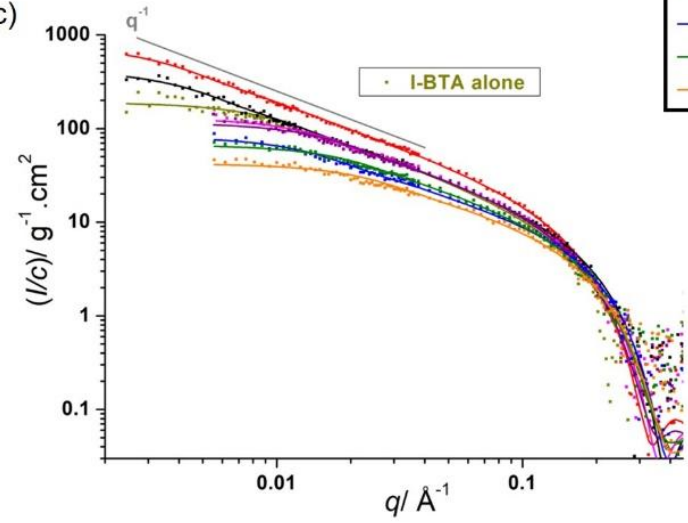

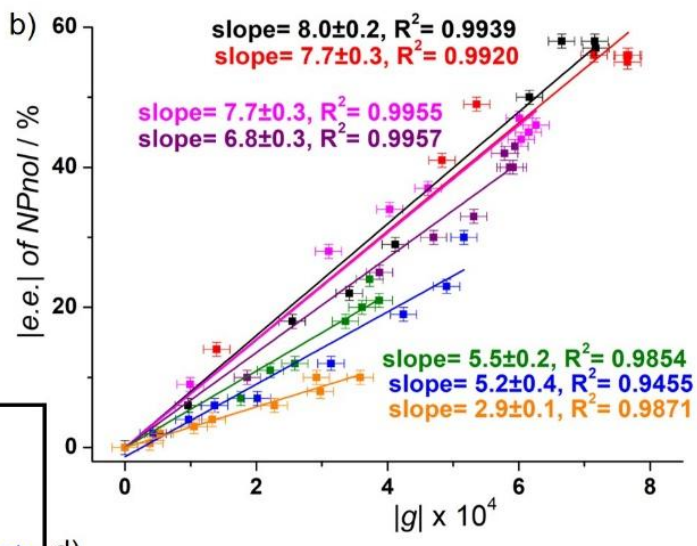

d)

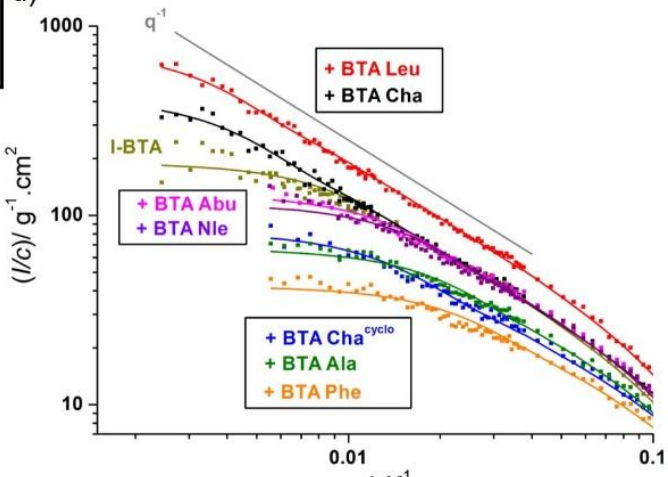

$q / A^{-1}$

Figure 2 Probing the influence of the sergeant on the properties and structure of catalytic co-assemblies. (a) Plot of the enantioselectivity in NPnol and Kuhn anisotropy factor as a function of the fraction of sergeants in the mixtures. (b) Plot of the enantioselectivity versus the Kuhn anisotropy factor for all the S\&S-type mixtures mentioned in this study. The fitted lines for BTA Abu and BTA Leu mixtures are superimposed. BTA Cha and BTA (S)-Cha have been used for CD analyses and catalytic experiments, respectively. (c) SANS analyses: scattered intensity $\left(\mathrm{cm}^{-1}\right)$ normalized by the (total BTA) concentration for I-BTA alone (6.0 g. $\left.\mathrm{L}^{-1}, 8.6 \mathrm{mM}\right)$ and for the S\&S-type mixtures containing I-BTA $\left(4.0 \mathrm{~g} \cdot \mathrm{L}^{-1}, 5.8 \mathrm{mM}\right)$ and the different sergeants $\left(3.7 \pm 0.1 \mathrm{mM}, \mathrm{f}_{\mathrm{s}}{ }^{0}=39 \%\right)$ in $\mathrm{C}_{7} \mathrm{D}_{8} . \mathrm{Analysis}$ is shown on the full scattering vector range. (d) Same analyses than (c) but below $q=0.1 \AA^{-1}$ to highlight the differences between the scattering curves at low $q$ values.

Scrutinizing the correlation plots in Figure $2 b$ further stresses out the following intriguing points: i) ee and $g$ values level off at non optimal values for BTA Abu and BTA Nle even though the slope of the plot for these sergeants is similar to that of BTA Cha and BTA Leu, i.e. the best sergeants, and ii) the slopes for BTA Cha ${ }^{\text {cyclo, }}$ BTA Ala and BTA Phe are significantly lower than those exhibited by the other sergeants. The latter point infers that a direct prediction of the selectivity (relatively to $e e_{\max }$ ) based solely on the optical purity of the supramolecular helices is not possible in the present system and that other parameters have to be taken into consideration to fully rationalize the correlation plots of Figure $2 \mathrm{~b}$.

\section{Structural characterization of the S\&S co-assemblies}

We performed SANS, FT-IR and variable-temperature (VT) CD analyses of the soldier and sergeants, individually, and of their mixtures in order to unveil the structural features of the coassemblies. Analyses have been performed with copper (FT-IR, VT-CD) or without copper (SANS) but its coordination to I-BTA is not expected to significantly influence the structure of the coassemblies as found previously in the case of I-BTA/BTA Cha mixtures. ${ }^{[8]}$ The soldier, I-BTA, was previously found to assemble on its own through a cooperative mechanism in toluene $^{[8 \mathrm{~b}]}$ under the form of stacks. Fitting its scattering curve (Figure 2c) with the form factor for cylindrical objects having a circular cross-section of radius $11 \AA^{[8 c]}$ and a finite length now indicates that the stacks of I-BTA are relatively long $(I=42 \pm 2 \mathrm{~nm}$, $\mathrm{DP}_{\mathrm{n}}=60 \pm 3$ ) at $8.6 \mathrm{mM}$ in $\mathrm{C}_{7} \mathrm{D}_{8}$. Most ester BTAs investigated to date are known to self-assemble under the form of dimers ${ }^{[17]}$ and this is actually the case of all the sergeants investigated in this study at the concentrations engaged in the S\&S-type mixtures $(3.7 \pm 0.1 \mathrm{mM})$ as demonstrated by SANS (Figure S4) and FT-IR analyses (Figures S6 and S7). Subsequently, mixtures of I-BTA $(5.8 \mathrm{mM})$ and the different sergeants $\left(\mathrm{f}_{\mathrm{s}}{ }^{0}=39 \%\right)$ have been analysed by SANS and the results are shown in Figure $2 \mathrm{c}$ (note that the scattered intensity is normalized by the total BTA concentration). The significant differences in scattered intensity at intermediate and low $q$ values between the different mixtures are a first indication of the different nature of the co-assemblies in terms of composition and length.

Previous studies of a mixture between I-BTA and BTA Cha indicated partial co-assembly, i.e. that a part of BTA Cha molecules incorporate into the stacks of I-BTA while the other part remains as dimers. ${ }^{[8 \mathrm{~b}, 8 \mathrm{c}]}$ Accordingly, SANS data for the different S\&S-type mixtures in Figure $2 c$ were modelled by a combination of rigid cylinders of finite length (consisting of I-BTA and a fraction of the sergeant) and spheres (consisting of the sergeant only). The radius of both cylinders and spheres was fixed to $11 \pm 1 \AA$ which is consistent with the value extracted by fitting their individual SANS data (Figure S4). ${ }^{[8 c]}$ The only adjustable parameters were the proportion of sergeants that co- 
assemble with I-BTA into cylinders $\left(\mathrm{f}_{\mathrm{s}}{ }^{\mathrm{s}}=\right.$ fraction of sergeants in stacks) and the length (I) of these cylinders. The quality of the fits allows a precise determination of these parameters (Table 1). ${ }^{[19]}$

Table 1. Geometrical features of the co-assemblies determined by fitting the SANS data (BTA in stacks, $\mathrm{fs}^{\mathrm{s}}, \mathrm{I}$ and $\mathrm{DP}_{\mathrm{n}}$ ) and FT-IR data (fs ${ }^{\mathrm{s}} \mathrm{IR}$ ) of S\&S-type mixtures $\left(f_{s}^{0}=39 \%\right)$.

\begin{tabular}{ccccc} 
sergeant & $\begin{array}{c}\text { BTA in } \\
\text { stacks } \\
(\%)^{[\mathrm{b}]}\end{array}$ & $\begin{array}{c}\mathrm{f}_{\mathrm{s}}{ }^{\mathrm{s}}(\%)^{[\mathrm{c}]}, \\
\mathrm{f}_{\mathrm{s}}{ }^{\mathrm{s}}-\mathrm{IR}(\%)^{[\mathrm{c}],[\mathrm{d}]}\end{array}$ & $\mathrm{I}(\mathrm{nm})^{[\mathrm{e}]}$ & $\mathrm{DP}_{\mathrm{n}}{ }^{[\mathrm{f}]}$ \\
\hline none ${ }^{[\mathrm{a}]}$ & 100 & - & $42 \pm 2$ & $60 \pm 3$ \\
BTA Leu & $94 \pm 6$ & $35 \pm 4,36 \pm 1$ & $120 \pm 10$ & $170 \pm 15$ \\
BTA Cha & $85 \pm 1$ & $29 \pm 2,28 \pm 1$ & $100 \pm 10$ & $143 \pm 15$ \\
BTA Abu & $93 \pm 7$ & $35 \pm 4,23 \pm 2$ & $28 \pm 2$ & $40 \pm 3$ \\
BTA Nle & $85 \pm 15$ & $29 \pm 10,20 \pm 2$ & $25 \pm 2$ & $38 \pm 2$ \\
BTA Cha ${ }^{\text {cyclo }}$ & $68 \pm 5$ & $11 \pm 6,7 \pm 2$ & $30 \pm 5$ & $43 \pm 7$ \\
BTA Ala & $68 \pm 9$ & $12 \pm 10,2 \pm 2$ & $20 \pm 1$ & $29 \pm 1$ \\
BTA Phe & $61 \pm 1$ & $0.5 \pm 3,2 \pm 2$ & $17 \pm 1$ & $24 \pm 1$ \\
\hline
\end{tabular}

[a] I-BTA alone. [b] Molar fraction of BTA in stacks $=([$ I-BTA $]+[$ sergeant in stacks]) / (total BTA concentration). The minimal fraction of BTA in stacks= fraction of $\mathbf{I - B T A}=1-\mathrm{f}_{\mathrm{s}}{ }^{0}=61 \%$. [c] Fraction of sergeants in stacks= [sergeant in stacks] / ([I-BTA] + [sergeant in stacks]). $\mathrm{f}_{\mathrm{s}}{ }^{\mathrm{s}} \max =\mathrm{f}_{\mathrm{s}}{ }^{0}=39 \%$ (full coassembly). [d] From FT-IR data. [e] Length of the stacks. [f] Number-average degree of polymerization assuming a stack intermolecular distance of $3.5 \AA$ and $\mathrm{DP}_{\mathrm{w}} / \mathrm{DP}_{\mathrm{n}}=2 .^{[20]}$

The geometrical features of the co-assemblies as extracted from the SANS data differ strongly according to the nature of the sergeant. In the cases of BTA Leu and BTA Cha, the major part of the sergeants co-assemble and few sergeants remain as dimers. Interestingly, these co-assemblies are longer $\left(D_{n}>140\right)$ than the self-assemblies of I-BTA suggesting a favorable free energy of co-association between these two monomers. A high fraction of BTA Abu and BTA Nle monomers also co-assemble with I-BTA but the length of the resulting co-assemblies are shorter $\left(D P_{n} \approx 40\right)$ than the self-assemblies of the soldier. Finally, for S\&S-type mixtures with BTA Cha ${ }^{\text {cyclo }}$, BTA Ala or BTA Phe, the fraction of the sergeants incorporated into the stacks is low $\left(\mathrm{f}_{\mathrm{s}}{ }^{\mathrm{s}}<<\mathrm{f}_{\mathrm{s}}{ }^{0}\right)$ and co-assemblies are short $\left(\mathrm{DP}_{\mathrm{n}}<43\right)$.

FT-IR analyses are informative about the nature of the hydrogen-bonded species present in these S\&S-type mixtures (Figure S5). Ester-bonded dimers formed by the sergeants and amide-bonded stacks formed upon co-assemblies can be distinguished ${ }^{[21]}$ which allow for an independent determination of the $\mathrm{fs}^{\mathrm{s}}$ values. These $\mathrm{fs}^{\mathrm{s}}$ values are in good agreement with those determined by SANS analyses (compare $\mathrm{fs}^{\mathrm{s}}$ and $\mathrm{fs} \mathrm{s}^{\mathrm{s}}-\mathrm{IR}$ in Table 1). Simulated FT-IR spectra constructed by making the same assumption than for fitting the SANS data, i.e. that mixtures are composed exclusively of sergeant dimers and mixed stacks, are in good agreement with the experimental ones (Figures S6 and S7). Notably, in the case of S\&S-type mixtures with BTA Leu, BTA Cha, BTA Nle and BTA Abu, the amount of $\mathrm{N}-\mathrm{H}$ bonded to amide carbonyl increases significantly relatively to that of pure I-BTA confirming that these sergeant intercalate into the stacks of I-BTA by changing their hydrogen bonding pattern form ester-bonded (in dimer) to amide-bonded (in mixed stacks). Conversely, for mixtures with BTA Cha ${ }^{\text {cyclo }}$, BTA Ala and BTA Phe, a large amount of $\mathrm{N}-\mathrm{H}$ are bonded to ester carbonyl which translates into remarkably low $\mathrm{fs}^{\mathrm{s}}$ values $\left(\mathrm{fs}^{\mathrm{s}}\right.$-IR $\leq$ $7 \%$ ). For these mixtures, stack ends are detected whose amount nicely fits the $\mathrm{DP}_{\mathrm{n}}$ values extracted from the SANS data (Figure S7).

Finally, VT-CD analyses (Figure S8) were conducted for the S\&S-type mixtures with BTA Leu, BTA Nle and BTA Phe $\left(\mathrm{fs}^{0}=\right.$ $39 \%)$. The transition temperature for I-BTA coordinated to copper has been probed by VT-UV Vis analyses. The temperature at which the induced $C D$ band starts to appear (elongation temperature) is comparable for the three S\&S-type mixtures and is also similar to the transition temperature obtained by UV-Vis for the stacks of I-BTA.Cu. The co-assembly formation is more cooperative in the following order BTA Leu> BTA Nle> BTA Phe which is consistent with the formation of longer objects in the same order. Based on these CD data and the literature ${ }^{[15 a, 23 \mathrm{~m}]}$ the sequestration of I-BTA monomers by the sergeants, i.e. a competing role of the sergeant, does not occur to a significant amount in these systems since it would have led to a large decrease of the transition temperature relatively to the homoassemblies of the soldier.

These set of analyses conducted on the co-assemblies allow to determine that again the sergeants can be sorted into three main categories. The first category encompasses BTA Cha and BTA Leu that co-assemble favorably with the soldier and generate very long assemblies. Both sergeants can be considered as pure intercalators, with BTA Leu being slightly more efficiently incorporated into the stacks of I-BTA than BTA Cha. BTA Abu and BTA Nle belong to a second category given that they co-assemble with the soldier but yield moderately long helices. Our data suggest that a large fraction of these sergeants are intercalated into the stacks and that a tiny fraction is located at their extremities and plays the role of end cappers. ${ }^{[22]}$ Finally, the third category of sergeants, constituted of BTA Ala, BTA Cha ${ }^{\text {cyclo }}$ and BTA Phe, yields short assemblies embedding few sergeants. Here, we must stress out that a precise distinction of the role of these sergeants between chain cappers ${ }^{[15 b, 23]}$ and competitors, ${ }^{[15 a]}$ in which the sergeant decreases the length of I-BTA stacks by capping or by sequestrating I-BTA monomers, respectively, is challenging. However, for BTA Phe, the DP $_{n}$ value of 24 extracted from SANS data at low $q$ translates into a fraction of capper in stacks of $1 / 24=4 \%$ if all chain-ends are BTA Phe molecules. This value is of the same order of magnitude, but larger than the experimentally determined value of $\mathrm{f}_{\mathrm{s}}{ }^{\mathrm{s}}$ of BTA Phe. The SANS data thus reveals that co-assemblies contain either one or zero BTA Phe molecule per supramolecular polymer chain. Together with the aforementioned VT-CD data, this goes in favor of a capping role of BTA Phe. In the case of BTA Ala and BTA $\mathrm{Cha}^{\text {cyclo }}$, the experimentally determined value of $\mathrm{f}_{s}{ }^{\mathrm{S}}$ is larger than $1 / D P_{n}$ (but close to it), showing that their effect is mainly that of a chain capper with possibly some intercalation, even though again sequestration of a part of I-BTA monomers by these sergeants cannot be fully discarded based on the present data. Interestingly, the three categories established upon structural characterization of the co-assemblies corresponds to the three classes determined by the aforementioned combination of catalytic experiments and CD analyses.

\section{Refining the role of the sergeant in supramolecular helical catalysts}


Our previous studies of S\&S-type helical catalysts, based on a single sergeant, highlighted the correlation between the

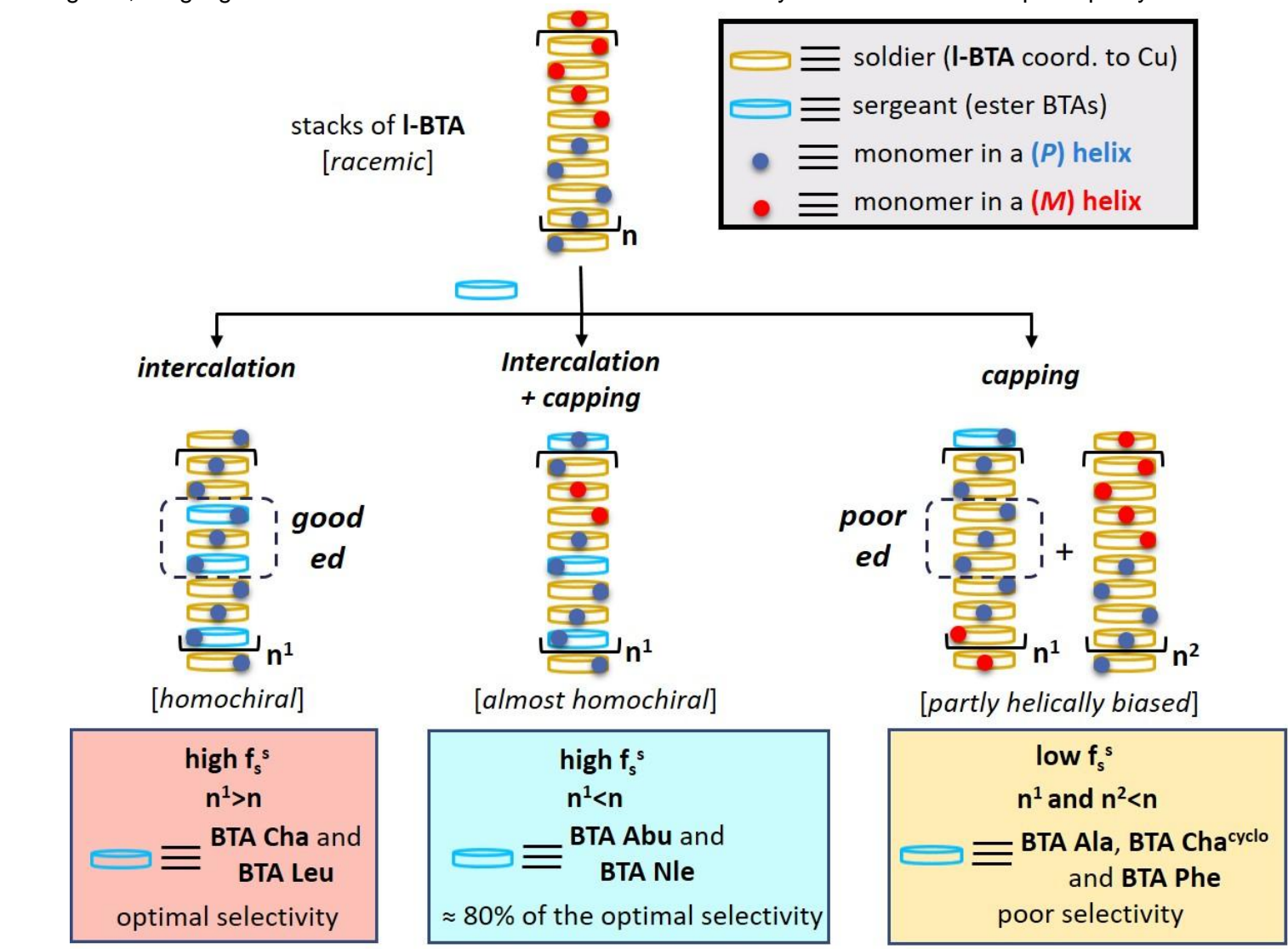

enantioselectivity (from $0 \%$ ee to optimal selectivity) provided in a catalytic reaction and the optical purity of the helices. In the

Scheme 2 Schematic representation of the role of the sergeant in determining the structure and selectivity of supramolecular helical catalysts composed of I-BTA (coordinated to copper) and different types of sergeants. The fraction of sergeants that do not incorporate into the stacks and remain as dimers is not represented. Likewise, only the predominant species (mixed stacks) are represented. The presence of other species present in minority, notably in the case of sergeants acting as cappers, cannot be ruled out. Triads highlighted in the dashed rectangles have the same helicity but differ by their composition: the central I-BTA is surrounded by sergeants (resp. by soldiers) for class a (resp. for class $c$ ). Their respective Cu complexes exhibit different levels of enantiodiscrimination (ed) in the course of the asymmetric reaction: good and poor for sergeants of class $a$ and class $c$, respectively.

present study, the characterization of catalytic S\&S-type mixtures composed of different types of sergeants allows to precise the role of the sergeant. The different roles exhibited by the sergeants and their influence on the structure and selectivity of the helical catalysts can be schematically represented as shown in Scheme 2 and described as follows:

- Class a (BTA Cha and BTA Leu): Very efficient coassembly with I-BTA occurs and favourable interactions between these sergeants and the soldier enable the formation of very long helices. In this case, the sergeant behaves as an intercalator. ${ }^{[15,23 \mathrm{~m}]}$ Homochiral helices are present for $\mathrm{f}_{\mathrm{s}}{ }^{0} \geq 39 \%$ and $f_{s}{ }^{0} \geq 50 \%$ with BTA Leu and BTA Cha mixtures, respectively. Homogeneous distribution of the ligand and the sergeants within these long co-assemblies generates an optimal chiral environment for the catalytic sites which in turn provides the optimal selectivity in the catalytic reaction of reference.

- $\quad$ Class $b$ (BTA Nle and BTA Abu): These sergeants also co-assemble efficiently with I-BTA but partly act as chain cappers $^{[15 b, 23]}$ leading to moderately long helices $\left(\mathrm{DP}_{\mathrm{n}} \approx 40\right.$ for $\mathrm{f}_{\mathrm{s}}{ }^{0}=39 \%$ ). The fact that these helices are not perfectly homochiral, even though the fraction of sergeants in stacks is important, might be explained either by the fact that the number of sergeants per stacks is insufficient to fully bias the assemblies (i.e. their correlation length is too short) and/or by the fact that the sergeants present at the chain ends (chain cappers) do not impose their helicity as efficiently as those within the chains (intercalators). Increasing the amount of the sergeant in the coassemblies also favours chain capping and as such both effects may compensate for $f_{s} \geq 39 \%$ preventing the formation of homochiral helices. The slopes of the correlation curves in Figure 2 indicate that the catalytic sites in BTA Nle and BTA Abu co-assemblies are as selective as those present in helices containing BTA Leu and BTA Cha. Therefore, the lower enantioselectivity provided by the respective helical catalysts likely stems from the lower helicity (presence of fragments of opposite handedness) of the co-assemblies. In other words class $a$ and $b$ sergeants impose the same local chiral environment on the catalytic sites, but fewer catalytic sites have the preferred helicity in the case of class $b$.

- Class c (BTA Cha ${ }^{\text {cyclo }}$, BTA Ala, BTA Phe): The ability of these sergeants to co-assemble with I-BTA is poor compared to that of the aforementioned sergeants. Intercalation of BTA Cha ${ }^{\text {cyclo }}$ and BTA Ala into the stacks is limited and the main role of BTA Phe is to act as a pure chain capper (vide supra). The modest handedness preference of these helices is related to the limited number of sergeants per stacks. For a similar overall chiral amplification (as indicated by $g$ values), the enantioselectivity provided by the catalytic sites of the different 
S\&S-type mixtures decreases in the following order: BTA Cha BTA Leu $\approx$ BTA Abu $\approx$ BTA Nle $>$ BTA Ala $\approx$ BTA Cha ${ }^{\text {cyclo }}>>$ BTA Phe (Figure $2 b$ ). This indicates that class $a$ et $b$ sergeants generate catalytic sites with similar enantioselectivities, but this is not the case for class $c$ sergeants. Short and sergeant-poor helices contain long sequences of I-BTA devoid of sergeants and even stacks of pure I-BTA are likely to be present in the case of S\&S-type mixtures with BTA Phe. It thus appears reasonable to consider that, even if both are positioned in single handed fragments, sergeant-rich sequences might be more selective than sergeant-poor ones. In a hypothetical triad in which I-BTA is sandwiched by two sergeants (see dashedrectangles in Scheme 2) the $\mathrm{Cu}$ complex and the sergeants will experience close contacts that can lead to a high degree of enantiodiscrimination in the course of the asymmetric reaction towards NPnol. These specific interactions are lacking in the co-assemblies composed of class $c$ sergeants where most ligands are actually surrounded by other ligands. This lack of short range contact with the sergeant could explain the lower enantioselectivities of the catalytic sites. It has been previously observed in hybrid metal complex - DNA conjugates that the base pair sequence in which the complex is intercalated greatly affects the selectivity of the catalytic reaction. ${ }^{[24]}$

It also important to note that modifications in the chemical structure of the sergeants leads to a drastic change in their coassembly behaviour. Even though elucidating the origin of the subtle effects leading to the divergent role played by the sergeants is out of the scope of the present study, it is quite remarkable to note that BTA Cha and BTA Phe have almost opposite co-assembly preference. Indeed, the former favours intercalation while the latter acts as a chain capper. Likewise, bulky and branched alkyl chains connected to the stereogenic centre are well tolerated and even tend to favour intercalation over capping (compare class $a$ and class $b$ sergeants, and catalytic results in Table S1). Finally, steric hindrance of the outer substituent also plays a role as highlighted by the lower capability of BTA Cha ${ }^{\text {cyclo }}$ to be incorporated into the stacks relatively to BTA Cha. Modification of the nature of the achiral ester side chain modulates the co-assembly behaviour of the sergeant and the selectivity of the corresponding helical catalysts.

\section{Conclusion}

In the present study, a set of sergeants-and-soldiers-type helical catalysts constructed on the same BTA assembling motif but differing by the nature of the groups present in the side chains of the sergeants have been characterized by a combination of $C D$, FT-IR and SANS experiments and correlated to catalytic data. Our results indicate that the enantioselectivity provided by these catalysts is directly proportional to the optical purity of the supramolecular helices if each sergeant is considered individually, but that the proportionality factor depends on the nature of sergeant. Accordingly, both the optical purity and the structure of the co-assemblies have to be considered to reach the optimal selectivity. Two sergeants were found to intercalate very efficiently in the stacks of the ligand generating very long assemblies which in turn provide the optimal selectivity in the reaction of reference. One sergeant acts as pure chain capper and despite biasing significantly the handedness of the helices it provides a comparatively lower extent of selectivity. The other sergeants behave in an intermediate manner, partly as intercalator and partly as chain capper, and provide a gradual evolution for the catalyst selectivity. This point sheds some light on the importance of the composition and length of the helices in the elaboration of a suitable chiral environment of the catalytic sites embedded into the helices. Not only the nature of the chemical groups attached to the stereogenic center but also the nature of the achiral moieties connected to the ester side chains will be considered as important elements for the design of the next generation of sergeants. A better understanding of the energetic pathways governing the role of the sergeants in these intricate co-assembly processes will also be crucial for the development of more efficient and more functional helical systems.

\section{Acknowledgements}

This work was supported by the Consejo Nacional de Ciencia y Tecnologia (CONACYT, post-doctoral fellowship of M. A. M.-A.) and the China Scholarship Council (CSC, PhD grant of Y.L.). Jacques Jestin (LLB, Saclay) is acknowledged for assistance with SANS experiment. The GDR 3712 Chirafun is acknowledged for allowing a collaborative network between the partners of this project. This manuscript is in honor of the 50 year anniversary of the French Polymer Group (Groupe Français des Polymères - GFP). Arianna Baldi is acknowledged for the synthesis and characterization of some precursors and BTA monomers.

Keywords: supramolecular chirality, supramolecular copolymerisation, helical catalyst, chirality amplification, sergeants-and-soldiers effect.

\section{References}

[1] M. M. Green, M. P. Reidy, R. J. Johnson, G. Darling, D. J. Oleary, G. Willson, J. Am. Chem. Soc. 1989, 111, 64526454.

[2] M. M. Green, J. W. Park, T. Sato, A. Teramoto, S. Lifson, R. L. B. Selinger, J. V. Selinger, Angew. Chem. Int. Ed. 1999, 38, 3139-3154.

[3] a) E. Yashima, K. Maeda, H. lida, Y. Furusho, K. Nagai, Chem. Rev. 2009, 109, 6102-6211; b) E. Yashima, N. Ousaka, D. Taura, K. Shimomura, T. Ikai, K. Maeda, Chem. Rev. 2016, 116, 13752-13990.

[4] a) L. J. Prins, P. Timmerman, D. N. Reinhoudt, J. Am. Chem. Soc. 2001, 123, 10153-10163; b) A. R. A. Palmans, J. A. J. M. Vekemans, E. E. Havinga, E. W. Meijer, Angew. Chem. Int. Ed. 1997, 36, 2648-2651; c) A. R. A. Palmans, E. W. Meijer, Angew. Chem. Int. Ed. 2007, 46, 8948-8968; d) L. Brunsveld, A. P. H. J. Schenning, M. A. C. Broeren, H. M. Janssen, J. A. J. M. Vekemans, E. W. Meijer, Chem. Lett. 2000, 292-293; e) L. Brunsfeld, B. G. G. Lohmeijer, J. A. J. M. Vekemans, E. W. Meijer, J. Incl. Phenom. Macro. 2001, 41, 61-64; f) M. H. Liu, L. Zhang, T. Y. Wang, Chem. Rev. 2015, 115, 7304-7397. 

Bouteiller, M. Raynal, Chem. Eur. J. 2016, 22, 3985-3990.

[6] S. H. Jung, J. Jeon, H. Kim, J. Jaworski, J. H. Jung, J. Am. Chem. Soc. 2014, 136, 6446-6452.

[7] a) Y. X. Wang, X. J. Li, F. Li, W. Y. Sun, C. J. Zhu, Y. X. Cheng, Chem. Commun. 2017, 53, 7505-7508; b) D. Yang P. F. Duan, L. Zhang, M. H. Liu, Nature Commun. 2017, 8; C) L. K. Ji, Y. T. Sang, G. H. Ouyang, D. Yang, P. F. Duan, Y. Q. Jiang, M. H. Liu, Angew. Chem. Int . Ed. 2019, 58, 844-848; d) P. Y. Li, B. Z. Lu, D. X. Han, P. F. Duan, M. H. Liu, M. Z. Yin, Chem. Commun. 2019, 55, 2194-2197; e) A Mukherjee, S. Ghosh, Chem. Eur. J., DOI: 10.1002/chem.202002056.

[8] a) A. Desmarchelier, X. Caumes, M. Raynal, A. VidalFerran, P. W. N. M. van Leeuwen, L. Bouteiller, J. Am. Chem. Soc. 2016, 138, 4908-4916; b) J. M. Zimbron, X. Caumes, Y. Li, C. M. Thomas, M. Raynal, L. Bouteiller, Angew. Chem. Int. Ed. 2017, 56, 14016-14019; c) Y. Li, X. Caumes, M. Raynal, L. Bouteiller, Chem. Commun. 2019 55, 2162-2165; d) Y. Li, L. Bouteiller, M. Raynal, ChemCatChem 2019, 11, 5212-5226; e) Y. Li, A. Hammoud, L. Bouteiller, M. Raynal, J. Am. Chem. Soc. 2020, 142, 5676-5688.

[9] a) S. Cantekin, T. F. A. de Greef, A. R. A. Palmans, Chem. Soc. Rev. 2012, 41, 6125-6137; b) Y. Dorca, J. Matern, G. Fernández, L. Sánchez, Isr. J. Chem. 2019, 59, 869-880.

[10] C. Kulkarni, E. W. Meijer, A. R. A. Palmans, Acc. Chem. Res. 2017, 50, 1928-1936.

[11] a) J. J. van Gorp, J. A. J. M. Vekemans, E. W. Meijer, J. Am. Chem. Soc. 2002, 124, 14759-14769; b) J. van Gestel, P. van der Schoot, M. A. J. Michels, Macromolecules 2003, 36, 6668-6673; c) A. J. Wilson, J. van Gestel, R. P. Sijbesma, E. W. Meijer, Chem. Commun. 2006, 4404-4406; d) M. M. J. Smulders, I. A. W. Filot, J. M. A. Leenders, P. van der Schoot, A. R. A. Palmans, A. P. H. J. Schenning, E. W. Meijer, J. Am. Chem. Soc. 2010, 132, 611-619; e) M. M. J. Smulders, P. J. M. Stals, T. Mes, T. F. E. Paffen, A. P. H. J. Schenning, A. R. A. Palmans, E. W. Meijer, J. Am. Chem. Soc. 2010, 132, 620-626; f) A. J. Markvoort, H. M. M. ten Eikelder, P. A. J. Hilbers, T. F. A. de Greef, E. W. Meijer, Nature Commun. 2011, 2, 509; g) H. M. M. ten Eikelder, A. J. Markvoort, T. F. A. de Greef, P. A. J. Hilbers, J. Phys. Chem. B 2012, 116, 5291-5301; h) F. García, L. Sánchez, J. Am. Chem. Soc. 2012, 134, 734742; i) P. J. M. Stals, J. C. Everts, R. de Bruijn, I. A. W. Filot, M. M. J. Smulders, R. Martín-Rapún, E. A. Pidko, T. F. A. de Greef, A. R. A. Palmans, E. W. Meijer, Chem. Eur. J. 2010, 16, 810-821; j) T. Kim, T. Mori, T. Aida, D. Miyajima, Chem. Sci. 2016, 7, 6689-6694; k) Y. Li, L. Dubreucq, B. G. Alvarenga, M. Raynal, L. Bouteiller, Chem. Eur. J. 2019, 25, 10650-10661; I) E. E. Greciano, J. Calbo, J. Buendía, J. Cerdá, J. Aragó, E. Ortí, L. Sánchez, J. Am. Chem. Soc. 2019, 141, 7463-7472.

[12] J. Markvoort, J. Phys. Chem. B 2019, 123, 6627-6642; b) L. N. J. de Windt, C. Kulkarni, H. M. M. ten Eikelder, A. J. Markvoort, E. W. Meijer, A. R. A. Palmans,

Macromolecules 2019, 52, 7430-7438; c) J. A. Berrocal, M. F. J. Mabesoone, M. G. Iglesias, A. Huizinga, E. W. Meijer, A. R. A. Palmans, Chem. Commun. 2019, 55, 1490614909; d) A. Das, G. Vantomme, A. J. Markvoort, H. M. M. ten Eikelder, M. Garcia-Iglesias, A. R. A. Palmans, E. W. Meijer, J. Am. Chem. Soc. 2017, 139, 7036-7044.

[13] a) W. Zhang, W. S. Jin, T. Fukushima, T. Mori, T. Aida, J. Am. Chem. Soc. 2015, 137, 13792-13795; b) J. Kang, D. Miyajima, T. Mori, Y. Inoue, Y. Itoh, T. Aida, Science 2015, 347, 646-651.

[14] a) B. Adelizzi, A. Aloi, A. J. Markvoort, H. M. M. Ten Eikelder, I. K. Voets, A. R. A. Palmans, E. W. Meijer, J. Am. Chem. Soc. 2018, 140, 7168-7175; b) R. van Buel, D. Spitzer, C. M. Berac, P. van der Schoot, P. Besenius, S. Jabbari-Farouji, J Chem Phys 2019, 151, 014902.

[15] a) G. Vantomme, G. M. ter Huurne, C. Kulkarni, H. M. M. ten Eikelder, A. J. Markvoort, A. R. A. Palmans, E. W. Meijer, J. Am. Chem. Soc. 2019, 141, 18278-18285; b) E. Weyandt, G. M. ter Huurne, G. Vantomme, A. J. Markvoort, A. R. A. Palmans, E. W. Meijer, J. Am. Chem. Soc. 2020, 142, 6295-6303.

[16] B. Adelizzi, N. J. Van Zee, L. N. J. de Windt, A. R. A. Palmans, E. W. Meijer, J. Am. Chem. Soc. 2019, 141, 6110-6121.

[17] a) A. Desmarchelier, B. G. Alvarenga, X. Caumes, L. Dubreucq, C. Troufflard, M. Tessier, N. Vanthuyne, J. Idé, T. Maistriaux, D. Beljonne, P. Brocorens, R. Lazzaroni, M. Raynal, L. Bouteiller, Soft Matter 2016, 12, 7824-7838; b) A. Desmarchelier, M. Raynal, P. Brocorens, N. Vanthuyne, L. Bouteiller, Chem. Commun. 2015, 51, 7397-7400.

[18] Mixtures with a fraction of sergeant higher than $65 \%$ led, for some S\&S combinations, to viscous solutions that cannot be reliably analyzed.

[19] For the mixtures with BTA Cha, this adapted fitting procedure gives similar results to the one previously employed in reference [8c].

[20] T. F. A. De Greef, M. M. J. Smulders, M. Wolffs, A. P. H. J. Schenning, R. P. Sijbesma, E. W. Meijer, Chem. Rev. 2009, 109, 5687-5754.

[21] Except for BTA Ala (see Material and Methods in the SI).

[22] The role of BTA Nle in our study is somewhat different from that of the same sergeant in the study of Vantomme et al. (ref 15a) for which BTA Nle was found to play the role of intercalator (respectively sequestrator) when present in a low (respectively high) fraction relatively to the soldier. This is likely due to the differences between the 2 systems under study (nature of the soldier, solvent).

[23] a) R. P. Sijbesma, F. H. Beijer, L. Brunsveld, B. J. B. Folmer, J. H. K. K. Hirschberg, R. F. M. Lange, J. K. L. Lowe, E. W. Meijer, Science 1997, 278, 1601-1604; b) F. Lortie, S. B. Boileau, L. Bouteiller, C. Chassenieux, F. Laupretre, Macromolecules 2005, 38, 5283-5287; c) W. Knoben, N. A. M. Besseling, L. Bouteiller, A. C. Stuart, Phys. Chem. Chem. Phys. 2005, 7, 2390-2398; d) W. Knoben, N. A. M. Besseling, M. A. Cohen Stuart, Macromolecules 2006, 39, 2643-2653; e) W. Knoben, N. A. M. Besseling, M. A. C. Stuart, J Chem Phys 2007, 126, 
024907; f) T. Pinault, C. Cannizzo, B. Andrioletti, G. Ducouret, F. Lequeux, L. Bouteiller, Langmuir 2009, 25, 8404-8407; g) T. Pinault, B. Andrioletti, L. Bouteiller, Beilstein J. Org. Chem. 2010, 6, 869-875; h) M. M. J. Smulders, M. M. L. Nieuwenhuizen, M. Grossman, I. A. W. Filot, C. C. Lee, T. F. A. de Greef, A. P. H. J. Schenning, A. R. A. Palmans, E. W. Meijer, Macromolecules 2011, 44, 6581-6587; i) G. M. ter Huurne, P. Chidchob, A. G. Long, A. Martinez, A. R. A. Palmans, G. Vantomme, Chem. Eur. J. 2020, 26, 9964-9970; j) S. C. Karunakaran, B. J. Cafferty, M. Peláez-Fernández, K. Neselu, I. Schmidt-Krey, A. Fernandez-Nieves, G. B. Schuster, N. V. Hud, Polym. Chem. 2018, 9, 5268-5277; k) P. Besenius, J. Polym. Sci. Pol. Chem. 2017, 55, 34-78; I) S. C. Karunakaran, B. J. Cafferty, K. S. Jain, G. B. Schuster, N. V. Hud, Acs Omega 2020, 5, 344-349; m) E. Weyandt, M. F. J. Mabesoone, L. N. J. De Windt, E. W. Meijer, A. R. A. Palmans, G. Vantomme, Org. Mater. 2020, 2, 129-142.

[24] A. J. Boersma, J. E. Klijn, B. L. Feringa, G. Roelfes, J. Am. Chem. Soc. 2008, 130, 11783-11790. 


\section{Entry for the Table of Contents}

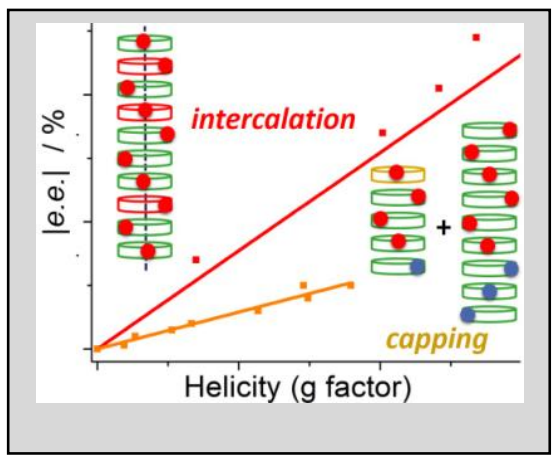

Co-assemblies between a benzene-1,3,5-tricarboxamide (BTA) ligand (soldier) and different types of enantiopure BTAs (sergeants) are evaluated as helical catalysts. The selectivity is related to the helicity and structure of the supramolecular helices (length, composition) which strongly depend on the role adopted by the sergeant in the co-assembly process (from intercalation to pure chain capping).

Institute and/or researcher Twitter usernames: @raynal_cnrs (M.R.) 